\title{
PREVALENCIA DE DEFICIENCIA DE VITAMINA A Y ANEMIA EN NIÑOS MENORES DE CINCO AÑOS DE PERÚ
}

\author{
Jaime Pajuelo ${ }^{1, a}$, Marianella Miranda ${ }^{2, b}$, Rosa Zamora ${ }^{3, a}$
}

\begin{abstract}
RESUMEN
Objetivos. Determinar la prevalencia de deficiencia de vitamina A (DVA) y anemia nutricional (AN), en menores de cinco años en Perú. Materiales y métodos. Estudio transversal con muestreo probabilístico, estratificado y multietápico realizado entre noviembre de 2007 y abril de 2010. Se incluyó 2736 niños para AN y 1465 para DVA. Se definió AN a valores de $\mathrm{Hb}<11 \mathrm{~g} / \mathrm{dL}$. La DVA fue identificada por retinol sérico con valores $<20 \mu \mathrm{g} / \mathrm{dL}$. Se estudiaron variables sociodemográficas relacionadas con el niño y la madre, además de su participación en programas de control de crecimiento y desarrollo, programa integral nutrición y suplementación con hierro y vitamina A. Se realizó el análisis para muestras complejas, se calculó estadísticas descriptivas y de regresión logística con un IC del 95\% y un nivel de significación de $p<0,05$. Resultados. La prevalencia de DVA fue de 11,7\% (IC 95\%: 9,4-14,4), las prevalencias más altas fueron en niños menores de cinco meses (44,6\%), y que viven en áreas rurales (19,5\%). La prevalencia de AN fue de 33\% (IC 95\%: 29,9-36,1), siendo mayor en los niños menores de 11 meses (68,2\%) e hijos de madres con 13 a 19 años de edad $(55,4 \%)$. Conclusiones. La DVA es un problema de salud pública que se mantiene, siendo los más afectados los niños que viven en las áreas rurales y en la selva. La prevalencia de AN muestra una ligera mejora. Es necesario mejorar la eficiencia e impacto de los programas de suplementación con vitamina A y hierro.
\end{abstract}

Palabras clave: Vitamina A; Anemia; Niño; Factores de riesgo (fuente: DeCS BIREME).

\section{PREVALENCE OF VITAMIN A DEFICIENCY AND ANEMIA IN CHILDREN UNDER FIVE YEARS OF AGE IN PERU}

\begin{abstract}
Objectives. To determine the prevalence of vitamin A deficiency (VAD) and nutritional anemia (NA) in children under five in Peru. Materials and methods. Cross-sectional study with a stratified and multi-staged probabilistic sample conducted between November 2007 and April 2010.2,736 children were included for NA and 1,465 children for VAD. NA was defined as $\mathrm{Hb}<11 \mathrm{~g} / \mathrm{dL}$.VAD was identified by serum retinol levels $<20 \mu \mathrm{g} / \mathrm{dL}$. Sociodemographic variables related to the child and the mother were studied in addition to their participation in growth control and development programs, integrated nutrition program and supplementation with iron and vitamin A. Analysis of complex samples was performed, descriptive statistics and logistic regression with $95 \%$ confidence intervals and a significance level of $p<0.05$ were calculated. Results. The prevalence of VAD was $11.7 \%(95 \% \mathrm{Cl}: 9.4$ to 14.4$)$. The highest prevalences were in children under five months $(44.6 \%)$ and those living in rural areas (19.5\%). The prevalence of NA was $33 \%(95 \% \mathrm{Cl}: 29.9$ to 36.1$)$ and was higher in children under 11 months $(68.2 \%)$ and children of mothers aged $13-19$ years old $(55.4 \%)$. Conclusions. VAD is a public health problem that remains, with the most affected children living in rural areas and in the jungle. The prevalence of NA shows a slight improvement. It is necessary to improve the efficiency and impact of programs that include supplementation of vitamin A and iron.
\end{abstract}

Key words: Vitamin A; Anemia; Child; Risk factors (source: MeSH NLM).

\section{INTRODUCCIÓN}

A nivel mundial se estima que aproximadamente 140 millones de niños en edad preescolar sufren de la deficiencia de vitamina A (DVA) anualmente ${ }^{(1)}$. La vitamina A es necesaria para mantener la diferenciación celular dentro de límites normales; desarrollar resistencia contra las infecciones, mantener la integridad de los epitelios; la producción de glóbulos rojos, mantener un buen registro visual y en el proceso de reproducción (2). Igualmente, la Organización Mundial de la Salud (OMS) refiere que existen 1,6 billones de anémicos en el mundo, de los cuales aproximadamente dos tercios son niños preescolares, principalmente en África y en el sudoeste asiático ${ }^{(3)}$.

En el Perú los problemas nutricionales que afectan a los niños en edad preescolar son principalmente la deficiencia de hierro (AN) y la DVA. El primer estudio nacional sobre AN

\footnotetext{
Instituto de Investigaciones Clínicas, Facultad de Medicina, Universidad Nacional Mayor de San Marcos, Lima, Perú.

Centro Nacional de Alimentación y Nutrición, Instituto Nacional de Salud, Lima, Perú.

Hospital Nacional Dos de Mayo, Lima, Perú.

Médico cirujano; ${ }^{\mathrm{b}}$ nutricionista

Recibido: : 03-09-14 Aprobado: 08-04-15
}

Citar como: Pajuelo J, Miranda M, Zamora R. Prevalencia de deficiencia de vitamina A y anemia en niños menores de cinco años de Perú. Rev Peru Med Exp Salud Publica. 2015;32(2):245-51. 
fue el realizado el año 1975, en el marco de la Evaluación Nutricional del Poblador Peruano (ENPPE) donde se encontró un 42,2\% deAN en niños menores de cinco años; siendo la selva el ámbito más afectado con el 57,2\% ${ }^{(5)}$. Posteriormente, el Centro Nacional de Alimentación y Nutrición (CENAN) del Instituto Nacional de Salud (INS) del Perú, con el apoyo del Instituto Nacional de Estadística e Informática, publicó regularmente información respecto a la prevalencia de la AN mostrando que esta deficiencia pasó del 57\% en 1996 a 37,7\% el $2010{ }^{(6)}$. Respecto a la DVA en niños menores de cinco años, estudios nacionales también realizados por el CENAN, identifican prevalencias de $19,2 \%$ en 1997 , y de $13 \%$ en el $2001^{(7)}$.

El objetivo del presente trabajo es evaluar la magnitud y determinantes de la deficiencia de vitamina A (DVA) y anemia nutricional (AN), en niños menores de cinco años en Perú.

\section{MATERIALES Y MÉTODOS}

El INS/CENAN llevó a cabo el Monitoreo Nacional de Indicadores Nutricionales (MONIN), noviembre 2007-abril $2010{ }^{(8)}$. Su método fue una encuesta continua (transversal repetida), por muestreo aleatorio multietápico, del universo de niños menores de cinco años, dividido en cinco ámbitos geográficos. Los procedimientos bioquímicos aplicados a los niños en cada hogar evaluado fueron: hemoglobina mediante el sistema HemoCue ${ }^{\circledR}(n=2736)$; mientras que retinol sérico se obtuvo a una parte de ellos ( $n=1465)$, a través de una muestra de sangre venosa del brazo.

Entre los criterios de exclusión (retinol y hemoglobina) para la toma de muestra sangre, se consideraron: a). Paciente convaleciente de alguna enfermedad $u$ operación; b). Paciente en estado febril mayor de $39^{\circ} \mathrm{C}$, en los 2 últimos días, y c). Paciente con diarrea (siete deposiciones diarias) en los dos últimos días.

En el caso del retinol sérico se obtuvo una muestra de $5 \mathrm{~mL}$ de sangre del brazo mediante la técnica de aguja libre. Dicha muestra fue centrifugada de manera manual por un tiempo no menor de diez minutos con la finalidad de separar el plasma del paquete de glóbulos rojos. El plasma de la muestra fue transferido a crioviales, tomando en consideración que este proceso se realice en ausencia de luz directa de día o luz solar, así como que la muestra mínima de plasma sea de 1,2 mL. Las muestras de plasma fueron conservadas en refrigeración con unidades de gel congeladas dentro de un coolerKST por un tiempo de cuatro horas como máximo. Luego de este tiempo las muestras de plasma fueron congeladas a una temperatura de $-20^{\circ} \mathrm{C}$, previa verificación de la temperatura con un termómetro digital. El transporte de las muestras hasta el laboratorio de CENAN se realizó con hielo seco (4 kg mínimo) o mediante el uso de un termo criogénico Dry Shipper. El punto de corte para determinar DVA fue $<20 \mu \mathrm{g} / \mathrm{dL}^{(9)}$.

Para el análisis de datos de hemoglobina, se consideró el punto de corte por debajo de $11 \mathrm{~g} / \mathrm{dL}$ como anemia ${ }^{(10)}$. Se realizó un ajuste de las mediciones de hemoglobina en función de la altitud ${ }^{(11)}$ de la que provenía la muestra.

Para la variable de control de crecimiento y desarrollo (CRED) se consideró al niño como "controlado" si presentaba más de 6 controles; esta información fue verificada en su carnet correspondiente. Con respecto a la enfermedad diarreica aguda (EDA) e infecciones respiratorias agudas (IRA), si el niño había presentado episodios de diarrea o tos persistente durante los 15 días previos a la encuesta, respectivamente.

En el caso del Programa Integral de Nutrición (PIN), se indagó por los miembros que están inscritos o son beneficiarios de cualquiera de los dos subprogramas (infantil - escolar). La respuesta inicialmente esperada fue la espontánea; si el entrevistado no recordaba se le leía la lista de programas existentes en la zona, o se describía sus características para que lo reconociera.

Se preguntó si el niño había recibido una dosis de sulfato ferroso durante la semana previa a la entrevista. Para la suplementación de vitamina $A$ el periodo de referencia fue en los últimos seis meses. En ambos casos la respuesta fue corroborada con el carnet correspondiente.

Se ha considerado pobres a los que presentan una o más necesidades básicas insatisfechas (NBI), y no pobres a los que las tenían satisfechas ${ }^{(12)}$. Para analizar la variable lactancia materna se preguntó a la madre del niño en investigación si alguna vez recibió pecho.

La información fue analizada mediante el paquete estadístico Statistical Pachage for the Social Sciences (SPSS) versión 15.0, con módulo Complex Samples. Los datos son presentados en prevalencias con su respectivo IC al 95\%. La asociación de las variables categóricas se hizo mediante la prueba de chi cuadrado con un $p<0,05$. En el caso de la normalidad se aplicó el test de KolgomorovSmirnov y para la homogeneidad de las varianzas el test de Levene. Se estimó análisis de regresión logística múltiple para verificar asociación entre factores determinantes. La presentación de los OR son ajustados.

El estudio se realizó en el marco de la vigilancia que periódicamente efectúa el Centro Nacional de Alimentación y Nutrición del INS. Se aplicó un 
consentimiento informado que formó parte del protocolo enviado al comité de investigación.

\section{RESULTADOS}

La Tabla 1 presenta la prevalencia de DVA encontrada, se observa que el $11,7 \%$ de niños presenta DVA. La prevalencia en el sexo femenino es de $12 \%$. En lo que respecta a la edad del niño, la mayor prevalencia se encontró en el grupo de 0 a 5 meses (44,6\%). La prevalencia disminuye conforme avanza la edad,

Tabla 1. Prevalencia de deficiencia de vitamina $A$ en niños menores de cinco años según características del niño y la madre

\begin{tabular}{|c|c|c|c|c|c|}
\hline & \multirow{2}{*}{$\mathbf{n}$} & Prevalencia & \multirow{2}{*}{ OR } & \multirow{2}{*}{ (IC 95\%) } & \multirow{2}{*}{$\begin{array}{c}\text { Valor } \\
\text { p }\end{array}$} \\
\hline & & (\%) (IC 95\%) & & & \\
\hline Prevalencia global & 1465 & $11,7 \quad(9,4-14,4)$ & & & \\
\hline \multicolumn{6}{|l|}{ Sexo } \\
\hline Masculino & 768 & $11,4(8,7-14,8)$ & 1,0 & & 0,589 \\
\hline Femenino & 697 & $12,0 \quad(9,1-15,6)$ & 1,1 & $(0,7-1,7)$ & \\
\hline \multicolumn{6}{|l|}{ Edad en meses } \\
\hline $0-5$ & 24 & $44,6(21,3-70,5)$ & 4,6 & $(0,1-17,1)$ & 0,049 \\
\hline $6-11$ & 92 & $17,4 \quad(9,3-30,1)$ & 1,3 & $(0,1-37,7)$ & \\
\hline $12-23$ & 274 & $12,8(8,4-19,5)$ & 1,9 & $(0,1-27,3)$ & \\
\hline $24-35$ & 338 & $8,7 \quad(5,9-12,6)$ & 1,5 & $(0,2-9,3)$ & \\
\hline $36-47$ & 381 & $13,8 \quad(9,8-18,9$ & 2,4 & $(0,9-6,3)$ & \\
\hline $48-59$ & 355 & $8,7 \quad(5,1-14,2)$ & 1,0 & & \\
\hline \multicolumn{6}{|l|}{ Gramos al nacer } \\
\hline $0-2499$ & 91 & $9,8 \quad(4,8-19,1)$ & 0,7 & $(0,3-1,7)$ & 0,444 \\
\hline $2500-3999$ & 1018 & $11,3(8,7-14,6)$ & 1,0 & & \\
\hline 4000 y más & 68 & $17,3(7,0-36,1)$ & 1,7 & $(0,6-4,5)$ & \\
\hline \multicolumn{6}{|l|}{ Edad de la madre } \\
\hline $13-19$ & 63 & $7,1 \quad(2,7-17,7)$ & 1,0 & & 0,129 \\
\hline $20-29$ & 671 & $13,9(10,7-18,0)$ & 2,4 & $(0,8-7.3)$ & \\
\hline $30-39$ & 546 & $8,4 \quad(6,0-11,6)$ & 1,5 & $(0,5-4.7)$ & \\
\hline $40-49$ & 140 & $10,2(5-19,9)$ & 3,5 & $(0,9-13.9)$ & \\
\hline Educación de la madre & & & & & 0,245 \\
\hline No lee ni escribe & 50 & $3,1 \quad(0,9-10,5)$ & 0,6 & $(0,1-4,4)$ & \\
\hline Primaria & 426 & $15,6(11,5-20,9)$ & 2,6 & $(0,8-8,4)$ & \\
\hline Secundaria & 633 & $10,9(7,9-14,8)$ & 2,0 & $(0,6-6,8)$ & \\
\hline Superior técnica & 202 & $7,7 \quad(4,1-13,8)$ & 1,7 & $(0,5-5,6)$ & \\
\hline Superior universitaria & 124 & $5,6 \quad(2,3-13,3)$ & 1,0 & & \\
\hline \multicolumn{6}{|l|}{ Área de residencia } \\
\hline Urbana & 1102 & $9,7 \quad(7,4-12,8)$ & 1,0 & & 0,008 \\
\hline Rural & 363 & $19,5(14,4-25,8)$ & 2,3 & $(1,2-4,3)$ & \\
\hline \multicolumn{6}{|l|}{ Metros de altitud } \\
\hline $0-999$ & 845 & $12,3(9,5-15,9)$ & 1,0 & & 0,609 \\
\hline 1000-2999 & 353 & $10,4 \quad(5,8-17,9)$ & 0,7 & $(0,2-1,9)$ & \\
\hline 3000 y más & 267 & $9,9(6,2-15,6)$ & 0,9 & $(0,2-4,9)$ & \\
\hline \multicolumn{6}{|l|}{ Ámbito geográfico } \\
\hline Lima Metropolitana & 282 & $9,4(5,4-15,9)$ & 1,0 & & 0,403 \\
\hline Resto costa & 324 & $12,5 \quad(8,3-18,4)$ & 1,6 & $(0,7-3,6)$ & \\
\hline Sierra urbana & 263 & $6,1 \quad(3,4-10,5)$ & 1,4 & $(0,3-6,9)$ & \\
\hline Sierra rural & 217 & $12,9(8,0-20,1)$ & 1,7 & $(0,3-11,4)$ & \\
\hline Selva & 379 & $16,2(11,6-22,2)$ & 2,6 & $(1,1-6,6)$ & \\
\hline \multicolumn{6}{|l|}{ Pobreza } \\
\hline No pobres & 861 & $10,0(7,3-13,6)$ & 1,0 & & 0,742 \\
\hline Pobres & 604 & $13,8(10,3-18,2)$ & 1,1 & $(0,7-1,8)$ & \\
\hline
\end{tabular}

salvo en el grupo de 36 a 47 meses. Se observa que la prevalencia de DVA es mayor conforme se presenta más peso al nacer. En cuanto a la edad de la madre, la prevalencia más alta de DVA fue en el grupo de 20 a 29 años con 13,9\%. Respecto al nivel educativo de la madre, la mayor prevalencia se encontró en el grupo de madres con educación primaria $(15,6 \%)$, aunque no debiéramos obviar a madres del grupo "no lee, ni escribe". Los niños de hogares pobres presentan mayor prevalencia 13,8\%; asimismo, aquellos que habitan entre 0 a los 999 metros de altitud (12,3\%) y los niños de la selva $(16,2 \%)$. Los niños que viven en áreas rurales $(19,5 \%)$, tienen mayor probabilidad de presentar DVA (OR 2,3; IC 95\%: 1,2-4,3).

En la Tabla 2, se presenta las prevalencias deficiencia de vitamina A según estrategias para promover la salud del niño y el control de la morbilidad. Se observa que la mayor DVA se encuentran en los niños que no tienen un control adecuado de CRED (12,8\%), que no son beneficiarios del PIN (12,2\%), que no recibieron suplementos de vitamina $A(12 \%)$ y que no recibieron suplementos de Hierro (11,8\%). En relación a indicadores de morbilidad, los niños que presentaron antecedente de EDA presentaron mayor DVA $(14,5 \%)$, lo mismo sucede con lo que tuvieron IRA (13\%). Por otro lado, el $15,1 \%$ de los niños que alguna vez tuvieron lactancia materna presentó DVA. Existe menor probabilidad de presentar DVA, en niños que no tuvieron antecedente de EDA (OR 0,6; IC 95\%: 0,3-0,9).

Tabla 2. Prevalencia de deficiencia de vitamina $A$ en niños menores de cinco años según estrategias en salud infantil y morbilidad del niño

\begin{tabular}{|c|c|c|c|c|c|c|}
\hline & $\mathbf{n}$ & $\begin{array}{l}\text { Pre } \\
(\%)\end{array}$ & $\begin{array}{l}\text { valencia } \\
\text { (IC 95\%) }\end{array}$ & OR & (IC 95\%) & $\begin{array}{c}\text { Valor } \\
p\end{array}$ \\
\hline \multicolumn{7}{|c|}{ Control CRED } \\
\hline Sí & 531 & 9,6 & $(6,7-13,5)$ & 1,0 & & \multirow{2}{*}{0,311} \\
\hline No & 931 & 12,8 & $(10,0-16,2)$ & 1,3 & $(0,8-2,2)$ & \\
\hline \multicolumn{7}{|c|}{ Beneficiario PIN } \\
\hline Sí & 221 & 8,4 & $(5,1-13,5)$ & 1,0 & & \multirow{2}{*}{0,129} \\
\hline No & 1244 & 12,2 & $(9,6-15,4)$ & 1,7 & $(0,9-3,3)$ & \\
\hline \multicolumn{7}{|c|}{ Suplemento de Vit. A } \\
\hline Sí & 95 & 6,0 & $(1,9-17,5)$ & 1,0 & & \multirow{2}{*}{0,067} \\
\hline No & 1370 & 12,0 & $(9,7-14,8)$ & 3,7 & $(0,9-14,9)$ & \\
\hline \multicolumn{7}{|c|}{ Suplemento de Hierro } \\
\hline Sí & 20 & 0 & - & & & \\
\hline No & 1445 & 11,8 & $(9,5-14,5)$ & & & \\
\hline \multicolumn{7}{|c|}{ Antecedente de EDA } \\
\hline Sí & 396 & 14,5 & $(10,1-20,4)$ & 1,0 & & \multirow{2}{*}{0,047} \\
\hline No & 1067 & 10,8 & $(8,4-13,7)$ & 0,6 & $(0,3-0,9)$ & \\
\hline \multicolumn{7}{|c|}{ Antecedente de IRA } \\
\hline Sí & 722 & 13,0 & $(10,2-16,6)$ & 1,0 & & \multirow[t]{2}{*}{0,762} \\
\hline No & 741 & 10,1 & $(7,7-13,3)$ & 0,9 & $(0,6-1,5)$ & \\
\hline \multicolumn{7}{|c|}{ Lactancia materna } \\
\hline No & 1083 & 10,1 & $(7,7-13,1)$ & 1,0 & & \multirow[t]{2}{*}{0,435} \\
\hline Sí & 365 & 15,1 & $(10,9-20,4)$ & 1,3 & $(0,7-2,7)$ & \\
\hline
\end{tabular}

Vit: vitamina, PIN: Programa Integral de Nutrición, CRED: control de crecimiento y desarrollo, EDA: enfermedad diarreica aguda, IRA: infecciones respiratorias agudas. 
La Tabla 3 presenta el número de niños a quienes se evaluó AN. La prevalencia nacional de AN fue 33,0\% existiendo mayor prevalencia en hombres $(34,7 \%)$. La prevalencia de AN fue mayor en el grupo de niños de 0 a 5 meses y el de 6 a 11 meses, ambas con 68,2\%. Conforme se incrementa la edad las prevalencias de AN van disminuyendo. En relación al peso al nacer del niño, la prevalencia más alta de AN la tienen los niños de más bajo peso al nacer. Los niños de madres adolescentes presentaron mayor prevalencia de AN $(55,4 \%)$, asimismo, los niños de madres sin ningún nivel de instrucción $(46,3 \%, p<0,034)$, que provienen

Tabla 3. Prevalencia de anemia nutricional en niños menores de cinco años según variables determinantes y su asociación

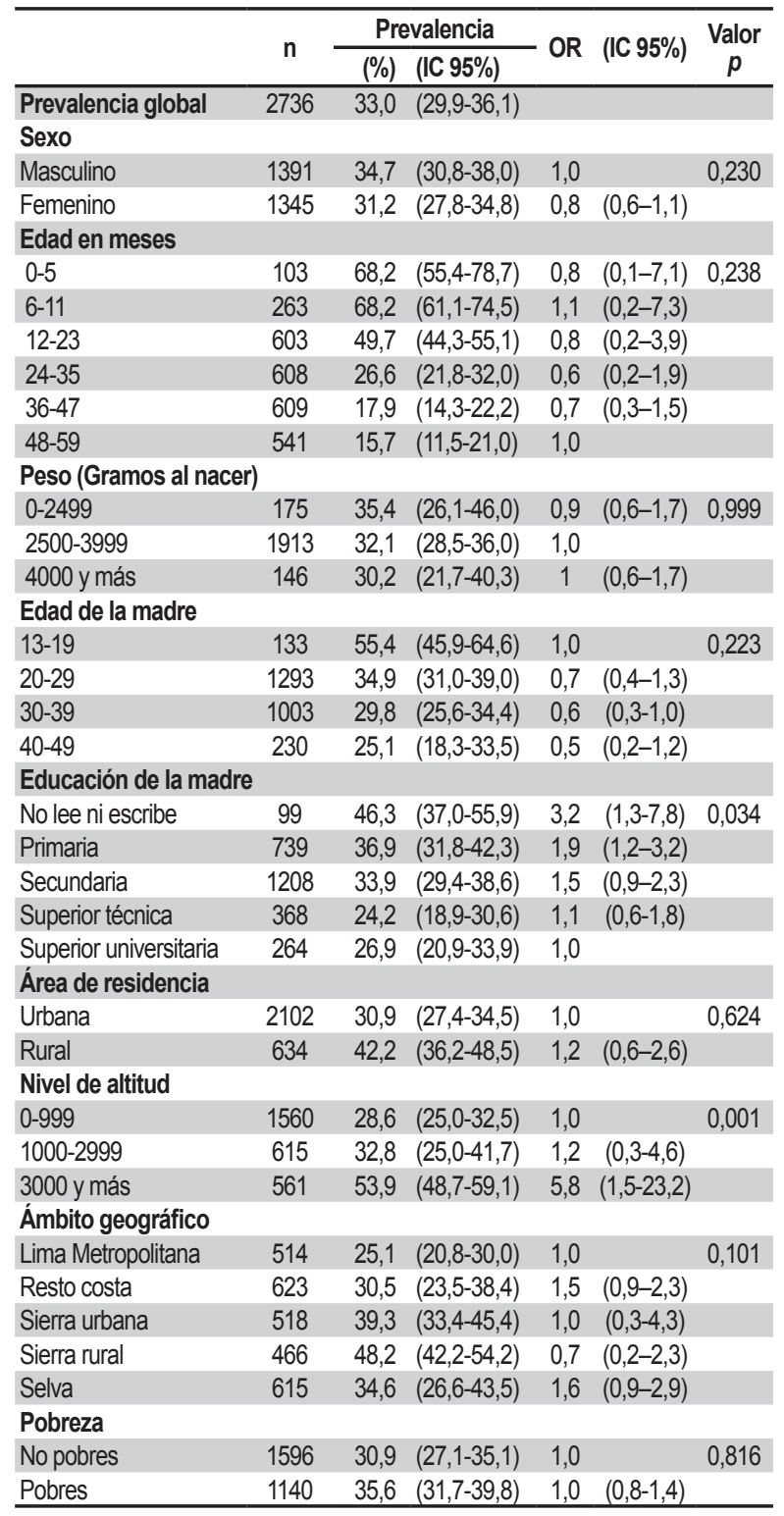

de hogares pobres (35,6\%), que viven en áreas rurales $(42,2 \%)$, que habitan por encima de los 3000 metros de altitud $(53,9 \%)$ y que residen en la sierra rural $(48,2 \%)$. Las variables que mejor explican el riesgo de presentar AN son: ser hijo de una madre que no lee ni escribe (OR 3,2, IC 95\%: 1,3-7,8) o con educación primaria (OR 1,9; IC 95\%: 1,2-3,2); y residir a un nivel de altitud de 3000 metros a más (OR 5,8; IC 95\%: 1,5-23,2).

La Tabla 4, muestra la relación de la AN con algunas estrategias que promueven la salud. Las mayores prevalencias se encontraron en los niños que no tenían un adecuado control CRED (33,3\%), en los beneficiarios del PIN (52\%), en los niños que recibieron suplementos con vitamina $A(43,6 \%)$, y niños que recibieron suplementos con hierro (44,6\%). Con respecto a los indicadores de morbilidad analizados, los niños que tuvieron antecedentes de EDA (41,8\%) e IRA (33\%), y los que habían recibido lactancia materna $(57,7 \%)$ presentaron mayor prevalencia de AN. Lactancia materna (OR 2,6; IC 95\%: 1,8-3,7) estuvo asociado con anemia.

Tabla 4. Prevalencia de anemia en niños menores de cinco años según variables determinantes relacionadas a estrategias en salud infantil y su asociación

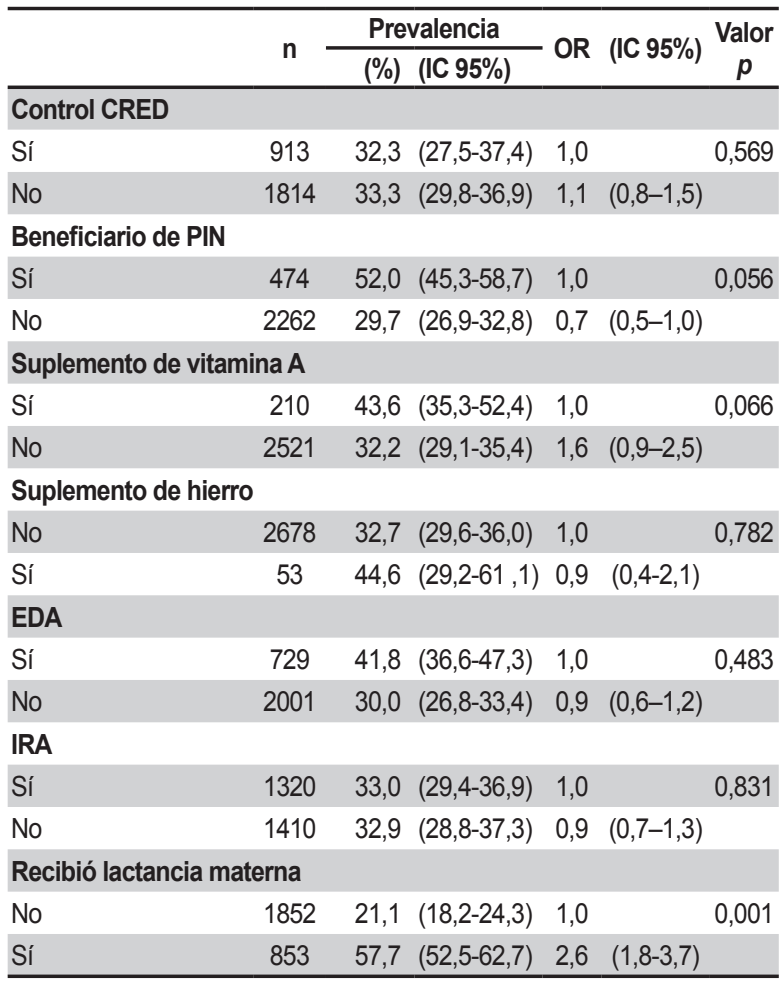

Vit: vitamina, PIN: Programa Integral de Nutrición, CRED: control de crecimiento y desarrollo, EDA: enfermedad diarreica aguda, IRA: infecciones respiratorias agudas. 


\section{DISCUSIÓN}

La prevalencia encontrada en DVA y AN en niños menores de cinco años del Perú, confirma su importancia como problema de salud pública. La causa de la AN es multifactorial, entre las más reconocidas están la deficiencia de micronutrientes (hierro, vitamina A, vitamina B12, folatos); infecciones (parasitosis, malaria, inmunodeficiencias) y desórdenes inherentes a las células de la serie roja. El objetivo de este estudio no fue identificar su causa principal, se asumió que esta deficiencia nutricional se explica principalmente por la deficiencia de hierro, siguiendo la conclusión de otros estudios ${ }^{(13)}$.

La OMS establece que la DVA es un problema de salud pública cuando la prevalencia de retinol sérico se encuentra por debajo del punto de corte considerado como adecuado $(20 \mu \mathrm{g} / \mathrm{dL})$. En ese sentido, y refiriéndose a niños mayores de 6 meses, cuando existe una prevalencia de $20 \%$ o más, el problema es intenso, de 10 a $19 \%$ es moderado y menos del $10 \%$ se trata de un problema leve ${ }^{(14)}$. El presente estudio muestra que la prevalencia de DVA es de $11,7 \%$, luego en el Perú se estaría frente a un problema moderado. Situación diferente en otros países de la región, por ejemplo Argentina, donde los niños de 5 a 33 meses de hogares carenciados, presentaban prevalencias de $26 \%$ (Buenos Aires), 32\% (Chaco) y $46 \%$ (Corrientes) ${ }^{(15)}$. De la misma manera, en Brasil alcanzó a $21,8 \%{ }^{(16)}$. Solo en Venezuela existe una prevalencia ligeramente menor $(10 \%)^{(17)}$. En la India, el problema es grave, la prevalencia pueden llegar a un $62 \%$ e incluso con presencia de signos clínicos como manchas de Bitot en un $0,8 \%{ }^{(18)}$.

En el Perú, uno de los primeros trabajos que reporta la situación de la DVA, fue realizado por el Instituto de Investigación Nutricional (IIN) en Piura $(32,8 \%)$ y Puno $(14,1 \%)$ el año $1991{ }^{(19)}$ A partir del año 1997, el país cuenta con información a nivel nacional 19,2\% (1997), $10,4 \%$ (1999), experimentándose un ligero incremento el $2001(13 \%)^{(7)}$. En lo que respecta a la localización del problema por ámbito geográfico, los estudios mencionados señalan que la sierra rural constituye el más afectado. El presente estudio confirma esta tendencia de la sierra rural (12,9\%), incorporándose la selva, con una prevalencia del $16,2 \%$.

Las principales causas de DVA pueden ser resumidas en dos categorías: (1) inadecuada alimentación, y (2) presencia de procesos infecciosos, que podrían incidir en una disminución de las concentraciones de retinol sérico en las primeras $24 \mathrm{~h}$ después de su instalación (en el caso EDA se agrega el aumento de la pérdida)(16).
No fue encontrada diferencia estadística significativa en relación a la prevalencia de DVA por sexo, resultado semejante fue observado en otros estudios (17-19), tampoco con la edad de la madre ${ }^{(16-20)}$.

Aquellos niños que no recibieron suplementación con vitamina $\mathrm{A}$, tuvieron mayor prevalencia de DVA, similares resultados se han encontrado en la India ${ }^{(18)}$ y en Guinea-Bissau ${ }^{(20)}$. Lo que llama la atención es que la cobertura de la suplementación es muy baja, lo que estaría indicando la existencia de algún problema en su operatividad y que por ende no cumpliría el objetivo para lo cual fue creado y por otro la presencia de DVA en los suplementados daría que pensar.

Existe abundante información a nivel nacional respecto a la AN ${ }^{(5,21)}$. Últimamente, el Monitoreo Nacional de Indicadores Nutricionales - (MONIN) del MINSA (20082009), señaló una prevalencia en niños de 6 a 35 meses del $43,4 \%$, siendo la sierra rural el área con más alta prevalencia $(58,1 \%){ }^{(22)}$. ENDES 2010 identificó una prevalencia de $37,7 \%{ }^{(6)}$. Los resultados del presente estudio, han mostrado que el $33 \%$ de niños presenta AN. Luego, y siguiendo la norma OPS, esta cifra indica que la AN constituye un problema moderado de salud pública en el Perú ${ }^{(13)}$, frente a las cifras de 1975 que mostraron que el problema era severo $\left(40 \%\right.$ y más) ${ }^{(21)}$. En este grupo de edad, existen tres factores que explicarían la prevalencia identificada: la reserva insuficiente de hierro al nacer, el bajo peso al nacer y la elevada velocidad de crecimiento, que da como resultado un balance negativo entre la ingesta y las pérdidas ${ }^{(23)}$.

En el mundo existen algunos países que, prácticamente, duplican la prevalencia peruana (Kenia con 78,8\% ${ }^{(24)}$, India con $75,3 \%{ }^{(25)}$, y $\left.67,7 \%{ }^{(18)}\right)$. También hay países con prevalencias ligeramente mayores como Palestina con $39,5 \%{ }^{(26)}$, Brasil $37 \%{ }^{(23)}$, y otros con prevalencias más bajas como en México 20,6\% (27) En Cuba, en el período del 2005 al 2011, la prevalencia pasó de 31,8 a $26 \%$, como consecuencia de una estrategia de fortificación de alimentos acompañado de intervenciones educativas ${ }^{(28)}$.

Este estudio muestra que los varones presentan una mayor prevalencia; esto podría deberse a que ellos serían más propensos a la $\mathrm{AN}$, consecuencia de su particular metabolismo del hierro (en absorción y pérdida), composición corporal e influencias hormonales ${ }^{(29)}$. Con relación a la edad, la prevalencia va disminuyendo conforme se incrementa la edad, fenómeno reportado en distintos estudios (24-26). La prevalencia encontrada en estos grupos se debería a insuficientes depósitos de este elemento para sostener el crecimiento de los niños de esta edad. La asociación 
encontrada con la educación de la madre ha sido reportada también en Kenia ${ }^{(24)}$.

En cuanto a la asistencia de los programas asistenciales, no se observa ninguna asociación entre las variables analizadas; lo mismo sucede con la morbilidad; se debe señalar, que existe una baja cobertura del programa de suplementación con hierro; en la India, pese a la existencia de un programa de este tipo para controlar la $\mathrm{AN}$, se ha constatado un incremento de su prevalencia ${ }^{(25)}$.

Los resultados reportados para con la lactancia materna y AN son confusos; muestran una mayor prevalencia y riesgo entre aquellos que sí la recibieron. Este hecho podría explicarse por el rango de edad del estudio. La LM exclusiva, hasta los seis meses de edad, asegura un correcto aporte de macro y micronutrientes; posteriormente, este requerimiento se incrementa y el niño requiere el aporte de otros alimentos, asociado al hecho de no presentar infecciones.

Da la impresión que los programas de asistencia para controlar la DVA y la AN no representarían una gran ayuda. Ello no debía llamar la atención; según la información registrada por ENDES 2010, la cobertura de ciertos programas dirigidos a mejorar la salud entre la población de 6 a 36 meses, presentaban bajas coberturas siendo del 35,4\% para CRED y 33,5\% para el PIN, suplementación con hierro 18,4\%. Lo mismo sucede con las intervenciones contra el EDA e IRA
(18,9 y $18,3 \%$, respectivamente). Si bien la estrategia más directa para atacar la $\mathrm{AN}$ es la suplementación con hierro, pareciera que la cobertura existente resulta demasiado baja, el $56,1 \%$ de los niños beneficiarios por el PIN fueron registrados como anémicos, cifra similar a lo encontrado en el presente trabajo ${ }^{(30)}$.

En conclusión, la prevalencia de AN muestra una ligera mejora con lo reportado previamente; sin embargo, estos progresos resultan aún insuficientes como lo indican todos los estudios realizados. En cuanto a la DVA, el problema se mantiene, siendo los más afectados los niños que viven en las áreas rurales y en la selva. Los programas de asistencia, no habrían contribuido a mejorar la problemática de la deficiencia de micronutrientes entre los niños, por ende, se recomienda estudios técnicos específicos que permitan mejorar la eficiencia e impacto de los programas de suplementación con vitamina A y hierro.

Contribuciones de autoría: JP y MM han participado en la concepción del artículo. JP estuvo a cargo de la redacción del artículo. MM obtuvo el financiamiento y participó en la recolección de datos y el análisis de datos. Además, RA contribuyó con la redacción. Los tres autores participaron en la aprobación de la versión final del artículo.

Fuentes de financiamiento: la ejecución ha sido financiada por el INS/CENAN. El diseño y análisis se han financiado con fondos de INS/CENAN.

Conflictos de interés: los autores declaran no tener conflictos de interés.

\section{REFERENCIAS BIBLIOGRÁFICAS}

1. Sommers A, Davidson FR, Annecy Accords. Assessment and control of vitamin A deficiency: the Annecy Accords. J Nutr. 2002 Sep;132(9 Suppl):2845S-2850S.

2. Sommer A, West KP. Vitamin A deficiency: Health, survival, and vision. New York: Oxford University Press; 1996.

3. World Health Organization. Worldwide prevalence of anaemia 1993-2005: WHO global database on anaemia. Geneva: WHO; 2008.

4. Olivares M, Walter T. Causas y consecuencias de la deficiencia de hierro. Rev Nutr. 2004;17(1):5-17.

5. Pajuelo J, Amemiya I. Anemia nutricional en la población infantil del Perú. Rev Med Per. 1992;347:51-5.

6. Instituto Nacional de Estadística e Informática. Encuesta Nacional Demográfica y de Salud Familiar 2010. Lima: INEI; 2010.
7. Instituto Nacional de Salud. Informe Nacional de deficiencia de vitamina A en niños menores de 5 años y mujeres en edad fértil 1997-2001. Lima: INS/ CENAN; 2004.

8. Instituto Nacional de Salud. Monitoreo Nacional de Indicadores Nutricionales (MONIN) [Internet]. Lima: INS/CENAN; 2008 [citado el 31 de marzo de 2015]. Disponible en: http://www.ins.gob. pe/repositorioaps/0/5/jer/resul_ moni_cenan/Protocolo_MONINversion\%202008.pdf

9. World Health Organization. Indicators for assessing vitamin A deficiency and their applications in monitoring and evaluating interventions programmes. Geneva: WHO; 1996.

10. World Health Organization. Catálogo ISBN 3-906412-33-4. Geneva: WHO; 2007.
11. Hurtado A, Merino C, Delgado E. Influence of anoxemia on haematopoietic activities. Arch Intern Med. 1945;75(5):284-323.

12. Instituto Nacional de Estadística e Informática. Mapa de Necesidades Básicas Insatisfechas de los Hogares a Nivel Distrital. Lima: INEI/UNFPA; 1994.

13. Kassebaum NJ, Jasrasaria R, Naghavi M, Wulf SK, Johns N, Lozano R, et al. A systematic analysis of global anemia burden from 1990 to 2010. Blood. 2014 Jan 30;123(5):615-24. doi: 10.1182/blood-2013-06-508325.

14. Organización Mundial de la Salud. Sistema de Información Nutricional sobre Vitaminas y Minerales [Internet]. Ginebra: OMS; 2011 [citado el 11 de febrero del 2015]. Disponible en: http://www.who.int/vmnis/database/ vmnis_upgrade/es/ 
15. Escobel N, Lejarraga H, Reybaud M, Picasso P, Lotero J, Pita de Portela $\mathrm{ML}$, et al. Déficit de vitamina A en una población infantil de alto riesgo social en Argentina. Rev Chil Pediatr. 2001;72(2):169-78.

16. Queiroz Dd, Paiva Ade A, Pedraza DF, Cunha MA, Esteves GH, Luna JG, et al. Vitamin A deficiency and associated factors in children in urban areas. Rev Saude Publica. 2013 Apr;47(2):248-56. doi: 10.1590/ S0034-8910.2013047002906.

17. De Abreu J, Borno S, Montilla M, Dini E. Anemia y deficiencia de vitamina A en niños evaluados en un centro de atención nutricional de Caracas. Arch Latinoam Nutr. 2005;55(3):226-34.

18. Laxmaiah A, Nair MK, Arlappa N, Raghu P, Balakrishna N, Rao KM, et al. Prevalence of ocular signs and subclinical vitamin A deficiency and its determinants among rural pre-school children in India. Public Health Nutr. 2012 Apr;15(4):568-77. doi: 10.1017/ S136898001100214X.

19. Del Águila R, López de Romaña $\mathrm{G}$, Brown K. Informe del proyecto Encuesta para determinar el nivel sérico de la vitamina $\mathrm{N}$ en niños menores de 6 años en 2 regiones del Perú. Lima: Instituto de Investigación Nutricional; 1991.

20. Danneskiold-Samsøe N, Bærent Fisker A, Jul Jørgensen M, Ravn H, Andersen A, Djogo Balde I, et al. Determinants of vitamin A deficiency in children between 6 month and 2 years of age in Guinea-Bissau. BMC Public Health. 2013;13:172-7.

21. Pajuelo R, Crespo I, Mendez C. Anemia nutricional en el Perú: un reto pendiente. Lima: Facultad de Medicina-UNMSM; 2007.

22. Instituto Nacional de Salud, Centro Nacional de Alimentación y Nutrición (CENAN). Monitoreo Nacional de Indicadores Nutricionales (MONIN) [Internet]. Lima: INS; 2009 [citado el 31 de marzo de 2015]. Disponible en: http://www.ins. gob.pe/repositorioaps/0/5/zop/ zona_izquierda_1/resumen ejecutivoMONIN2008-2009.pdf

23. Oliveira AP, Pascoal MN, Santos LC, Pereira SC, Justino LE, Petarli GB, et al. Prevalencia de anemia e sua associacao com aspectos sociodemográficos e antropométricos em criancas de Vitória, Esoirito Santo, Brasil. Cienc Saúde Colectiva. 2013;18(11):3273-80

24. Foote EM, Sullivan KM, Ruth LJ, Oremo J, Sadumah I, Williams TN, et al. Deteminants of anemia among preschool children in rural, Western Kenya. Am J Trop Med Hyg 2013;88 (4):757-764.

25. Pasricha SR, Black J, Muthayya S, Shet A, Bhat V, Nagaraj S, et al. Determinants of anemia among young children in rural India. Pediatrics. 2010 Jul;126(1):e140-9. doi: 10.1542/ peds.2009-3108.
26. Tsigga M, Grammatikopoulou MG. Assesing the silent epidemic of malnutrition in Palestina preschool children. J Epidemiol Glob Health. 2012 Dec;2(4):181-91. doi: 10.1016/j. jegh.2012.12.002.

27. De la Cruz-Góngora V, Villalpando S, Rebollar R, Shamah-Levy T, MéndezGómez Humarán I. Nutritional causes of anemia in Mexican children under 5 years. Results from the 2006 National Health and Nutrition Survey. Results from the 2006 National Health and Nutrition Survey. Salud Publica Mex. 2012 Mar-Apr;54(2):108-15.

28. Pita GM, Jiménez S, Basabe B, García RG, Macías C, Selva L, et al. Anemia in children under five years old in Eastern Cuba, 2005-2011. MEDICC Rev. 2014 Jan;16(1):16-23.

29. Chaparro CM. Setting the state for child health and development prevention on iron deficiency in early infancy. J Nutr. 2008 Dec;138(12):2529-33.

30. Ricaldi R. Servicio de procesamiento, análisis estadístico e informe técnico del análisis secundario de la base de datos de la ENDES; Producto No. 3: Informe Final. Lima; 2010.

Correspondencia: Marianella Yolanda Miranda Cuadros

Dirección: Calle Los Titanes 185 -Chorrillos Teléfono: 997320073

Correo electrónico:mymrirandac@gmail.com

\section{Consulte la versión electrónica de la Revista Peruana de Medicina Experimental y Salud Pública en} www.pubmed.gov

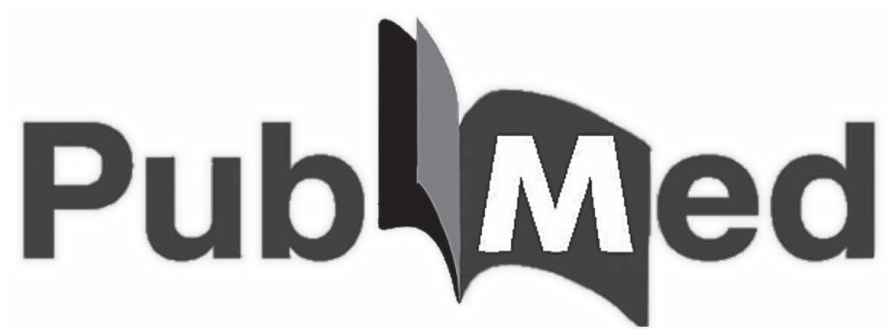

